\title{
Erratum to: Different efficacy of EGFR tyrosine kinase inhibitors and prognosis in patients with subtypes of EGFR-mutated advanced non-small cell lung cancer: a meta-analysis
}

Huan Wang · Jing Huang $\cdot$ Xiaojin Yu $\cdot$ Shuhua Han •

Xing Yan $\cdot$ Siqing Sun $\cdot$ Xiaoli Zhu

Published online: 19 September 2014

(C) Springer-Verlag Berlin Heidelberg 2014

Erratum to: J Cancer Res Clin Oncol

DOI 10.1007/s00432-014-1709-0

One of the affiliations of the first author was not included in the original publication. The correct information is given below.

The online version of the original article can be found under doi:10.1007/s00432-014-1709-0.

H. Wang $\cdot$ J. Huang $\cdot$ S. Han $\cdot$ S. Sun $\cdot$ X. Zhu $(\bowtie)$

Zhongda Hospital of Southeast University, Dingjiaqiao 87,

Gulou District, Nanjing 210009, China

e-mail: zhuxiaoli62@163.com

H. Wang $\cdot$ X. Yan

Medical School of Southeast University, Nanjing, China

$\mathrm{X} . \mathrm{Yu}$

Public Health School of Southeast University, Nanjing, China 